\title{
II. Der virtuelle Mengele
}

\section{Prototyp des SS-Arztes}

Die Zeugenaussagen, die die Frankfurter Staatsanwaltschaft vor allem in den 1960er und 1970er Jahren gesammelt hat und die Mengeles Verbrechen in Auschwitz dokumentieren, füllen fast drei Dutzend Aktenbände. ${ }^{1} 1981$ wurde auf der Grundlage dieser Zeugenaussagen ein neuer, deutlich erweiterter Haftbefehl erlassen. Auf 45 Seiten listet dieses Dokument neben Verbrechen, die Mengele im Rahmen seiner "Normaltätigkeit" als Lagerarzt in Auschwitz beging und solchen, die mit seinen pseudowissenschaftlichen Experimenten in Zusammenheng stehen, auch eine Vielzahl sogenannter „Exzeßtaten“ auf, die sich durch ihre extreme Grausamkeit auszeichnen. ${ }^{2}$ So habe er etwa, um nur zwei der bekanntesten Beispiele zu nennen, „den neugeborenen Säugling der Jüdin Sussmann aus Wien lebend ins Feuer geworfen“ oder ,in einer Mehrzahl von Fällen schwangere Frauen gezwungen [...], sich rücklings auf den Boden zu legen“, um diese dann mit seinen Stiefeln „so lange in den Bauch" zu treten, „bis der Abgang der Leibesfrucht eintrat“.3

Den Aussagen, die Mengele als derart sadistischen Täter schildern, der „aus Freude am Töten" 4 bestialisch gemordet habe, stehen eine Reihe von Zeugnissen gegenüber, die Mengele als „Mörder mit den weißen Handschuhen “5 charakterisieren, der sich niemals selbst die Finger schmutzig gemacht habe. Aus den in den Akten der Staatsanwaltschaft gesammelten Berichten ergibt sich so ein seltsam zwiespältiges Bild. 6 Bei der Bewertung der gesammelten Zeugenaussagen im Fall Mengele, dies sei einleitend gesagt, muß mit großer Sorgfalt vorgegangen werden. Ein Strafprozeß hat niemals stattgefunden, und die von der Staatsanwaltschaft gesammelten Aussagen konnten deshalb nie auf ihre Belastbarkeit überprüft werden. Dies soll nicht heißen oder auch nur andeuten, die Opfer hätten bewußt die Unwahrheit gesagt oder derartige Geschehnisse erfunden. Sicherlich kann man dem einen oder anderen Zeugen vorwerfen, er habe sich „wohl auch durch einen gewissen Geltungsdrang leiten lassen" und manche „Übertreibungen“ wird man auch als "hilflosen Versuch“ verstehen müssen, „das Grauen von Auschwitz" denen, die es nicht selbst erlebt haben, „mitzuteilen und verständlich zu machen“". Entschei-

1 Sta F/M, Az 4 Js 340/68, Erm.A., Bd. I-XXXIII.

2 Sta F/M, Az 4 Js, 340/68, Fahnd.A., Haftbefehl der 22. Strafkammer des Landgerichts Frankfurt am Main gegen Josef Mengele, 19. 1. 1981, S. 32-37, Zitate S. 32.

3 Ebenda, S. 34 und 36.

4 Ebenda, S. 32.

5 Zofka, KZ-Arzt, S. 259.

6 Vgl. ebenda, und Völklein, Mengele, S. 11-30.

7 Zofka, KZ-Arzt, S. 259, und Klee, Auschwitz, S. 476. 
dend aber dürfte sein, daß viele Josef Mengele zwanzig oder dreißig Jahre nach den Geschehnissen in Auschwitz schlicht mit anderen Wachmännern oder Lagerärzten verwechselten.

An dieser Stelle ist es hilfreich, einen vergleichenden Blick auf den Großen Frankfurter Auschwitz-Prozeß ${ }^{8}$ zu werfen. Auch dort konnte das Problem beobachtet werden, daß viele Zeugen Schwierigkeiten hatten, den einzelnen Angeklagten die jeweiligen Tatvorwürfe zuzuordnen, ein verständliches Problem vieler Auschwitz-Berichte: „Die Zeitzeugen verwechseln begreiflicherweise Daten, sie projizieren Taten auf Täter, die zwar sehr wohl Mörder waren, aber gerade diese Tat nicht begangen haben konnten." 9 Erklärlich wird dies, wenn man sich vor Augen führt, daß die Opfer zur Tatzeit meist völlig entkräftet waren und sich in Todesangst befanden; in einer solchen existentiellen Ausnahmesituation war es ihnen schlicht unmöglich, sich die Täter, denen sie ja häufig nur für wenige Sekunden gegenüberstanden, so einzuprägen, daß sie sie auch Jahrzehnte später noch zweifelsfrei identifizieren konnten. ${ }^{10}$ Anders verhielt es sich dagegen mit Zeugen, die auf Grund ihrer Funktion im Lager ständig, manchmal täglich mit dem Täter in Kontakt standen. Sie waren natürlich zu ungleich präziseren Aussagen fähig.

Im Fall Josef Mengele trifft dies vor allem auf die Häftlingsärzte und Pfleger zu, die gezwungen waren, mit ihm über einen langen Zeitraum hinweg zusammenzuarbeiten und die über Jahre und Jahrzehnte, oft unter Eid, ihre Aussagen ohne erhebliche Abweichungen wiederholten. ${ }^{11}$ Josef Mengele sei kein Sadist gewesen, so ihre weitgehend übereinstimmenden Angaben, „denn das Wesen eines Sadisten ist es ja, daß er an dem Schmerz seines Opfers Freude hat. Bei Mengele hatte man das Gefühl, daß er gar nicht merkt diesen Schmerz, der fällt ihm gar nicht auf. Sondern die Häftlinge waren für ihn Meerschweinchen, Ratten, mit deren Seelenleben man sich überhaupt gar nicht beschäftigt, [...] diese völlig distanzierte Haltung, die man beruflich zu seinem Material hat."12 Er habe die Häftlinge zu grausamsten Experimenten herangezogen, die er für wissenschaftlich gerechtfertigt hielt, und er habe Kranke und Schwache ohne Gnade selektiert, aber „körperlich mißhandelt hat er die Kranken nicht“13, „er machte sich die Hände nicht schmutzig"14. Nur gelegentlich habe er im Jähzorn einen Häftling geschlagen.

„Mengele ist nicht aus Mordlust in Auschwitz, sondern als Genetiker"15, so das Fazit Ernst Klees. Wenn er sich mit den Häftlingen befaßt, dann als Forscher und Wissenschaftler, nicht als Arzt. Eine Häftlingsärztin bestätigt, daß sie nie gesehen habe, „daß er einen Kranken untersuchte oder behandelte. Ich habe niemals in Birkenau einen deutschen Arzt gesehen, der einen Kranken behandelt hat." 16 Wie alle KL-Ärzte behandelte Mengele nicht - er selektierte für die Gaskammern. Von der

8 Vgl. S. $123 \mathrm{ff}$.

9 Klee, Auschwitz, S. 476.

10 Vgl. Völklein, Mengele, S. 30.

11 Vgl. ebenda.

12 So die Häftlingsärztin Ella Lingens, zit. nach: Zofka, KZ-Arzt, S. 260.

${ }_{13}$ Aussage Irena Bialowina, zit. nach Klee, Auschwitz, S. 487.

${ }^{14}$ Sta F/M, Az 4 Js 340/68, Erm.A., Bd. XIII, Bl. 3, Aussage Kazimierz Czelny, 30. 10. 1972.

Czelny war Hilfspfleger.

15 Klee, Auschwitz, S. 488.

${ }^{16} \mathrm{Sta}$ F/M, Az 4 Js 340/68, Erm.A., Bd. X, Bl. 100, Aussage Odette Abadi, 9. 6. 1972. 
Notwendigkeit dessen, was er tat, war er völlig überzeugt ${ }^{17}$, und einen jungen Arzt, den nach seiner ersten Selektion Wein- und Brechkrämpfe schüttelten, tröstete Mengele mit der Feststellung, ein Arzt an der Front müsse ebenfalls selektieren, denn mit der Festlegung der Reihenfolge der Operationen entscheide er oft über Leben und Tod der Soldaten. 18

Josef Mengele hat mit Sicherheit viele der Verbrechen, die in Auschwitz Alltagsverbrechen waren, begangen ${ }^{19}$ - besonders dann, wenn man seinen Anordnungen aus seiner Sicht keine oder ungenügend Folge leistete, ihn dadurch zu einem Zornesausbruch reizte und sich das "Geschehen" in seinem Zuständigkeitsbereich als Lagerarzt abspielte. ${ }^{20}$ So scheint die Schilderung einer Rampenselektion durchaus plausibel, in deren Verlauf Mengele eine Mutter, die sich nicht von ihrer dreizehnjährigen Tochter trennen wollte, zusammen mit ihrem Kind erschossen und anschließend „aus Wut über den Vorfall“21 den ganzen Transport ohne Ausnahme ins Gas geschickt haben soll. Auch daß Mengele während der Liquidierung des Zigeunerlagers im Sommer 1944 zwei Jungen „mit seinem Wagen zum Krematorium gefahren haben [soll], wo sie getötet wurden", ist durchaus glaubwürdig. Offenbar hatten sich die Kinder „während des Appells versteckt“, um dem Abtransport in die Gaskammern zu entgehen; dies hatte zur Folge, daß „die festgestellte Häftlingszahl nicht stimmte" 22 - was natürlich aus Sicht des Pedanten Mengele umgehend korrigiert werden mußte.

Exzeßtaten wie die oben beschriebenen Stiefeltritte gegen auf dem Boden liegende schwangere Frauen bis zum Abgang des Ungeborenen wollen dagegen zu dem Josef Mengele nicht recht passen, den die Häftlinge beschrieben, die durch ihre erzwungene Mitarbeit in engem Kontakt zu ihm standen. Es hat diese Taten in Auschwitz zweifellos gegeben - sie waren jedoch nicht Mengeles „Stil“. Das „Problem" Schwangerschaft etwa „löste“ er auf "elegantere“ Weise: Die werdende Mutter wurde für die nächste Selektion und damit für die Gaskammer vorgemerkt; alternativ wurde, wenn es dennoch zu einer Geburt gekommen war, der Säugling unmittelbar danach getötet - oft vor den Augen der Mutter und in der Regel durch eine Phenolinjektion ins Herz. Mengele tötete nicht aus Lust: Er erfüllte gnadenlos seine Aufgaben als Lagerarzt und selektierte für die Gaskammern - oder er opferte die Häftlinge dem Kalkül des hemmungslosen Forschers. ${ }^{23}$

17 Hans Münch im Gespräch mit Ulrich Völklein, nach: Völklein, Mengele, S. 14. Münch war Leiter der im Mai 1943 eingerichteten Hygienisch-Bakteriologischen Untersuchungsstelle der Waffen-SS und Polizei Süd-Ost nahe Auschwitz. Ähnlich Aurelia Wald, Blockälteste im Krankenbau, die betont, Mengele habe im Gegensatz zu manch anderen Ärzten „keinerlei Bedenken“ gehabt, die Häftlinge für die Gaskammern zu selektieren und sei „immer weit über die vorgeschriebene Zahl hinaus/gegangen]“. Sta F/M, Az 4 Js 340/68, Erm.A., Bd. III, Bl. 609-615, Aussage Aurelia Wald, 2. 11. 1959.

18 Aussage Hans Münch im Auschwitz-Verfahren, zit. nach: Klee, Auschwitz, S. 417.

19 Hans Münch umschrieb dies euphemistisch so: Mengele habe sich „dem Milieu von Auschwitz angepaßt“. Zit. nach: Zofka, KZ-Arzt, S. 260.

20 Ebenda, S. 261.

21 Sta F/M, Az 4 Js, 340/68, Fahnd.A., Haftbefehl der 22. Strafkammer des Landgerichts Frankfurt am Main gegen Josef Mengele, 19. 1. 1981, S. 15.

22 Ebenda.

23 Vgl. Zofka, KZ-Arzt, S. 261. 
Daß Mengele auch eine erhebliche Zahl von Exzeßtaten vorgeworfen wurde, bedarf einer Erklärung. Die grundsätzliche Möglichkeit von Verwechslungen ist, wie bereits geschildert, unter bestimmten Umständen bei Aussagen von AuschwitzOpfern gegeben. Mengele bot sich als Ziel einer solchen Verwechslung mit den tatsächlichen Peinigern geradezu an; um zu erklären, warum dies so war, soll zunächst nochmals der Frankfurter Auschwitz-Prozeß zum Vergleich herangezogen werden. In Verlauf der Verhandlung standen die Richter, wie oben angedeutet, vor ähnlichen Problemen. Bei solchen Tätern, die den Zeugen vom Namen oder der Erscheinung her geläufiger oder besonders bekannt waren, kam es im Vergleich zu weniger bekannten Tätern zu einer Anhäufung von teilweise ungerechtfertigten Tatvorwürfen. Außerdem wiederholten sich einige Beschreibungen besonders grausamer Mißhandlungen immer wieder. Das Treten schwangerer Frauen in den Bauch ist eines dieser Beispiele. Die Richter bezweifelten nicht, daß es in Auschwitz solche „Exzeßtaten" tatsächlich gegeben hat, doch die Häufigkeit der analogen, zum Teil jedoch unzutreffenden Vorwürfe führten das Gericht zu der Annahme, daß die unvorstellbare Grausamkeit tatsächlicher Einzeltaten für die Opfer zu einer Metapher für das unmenschliche und unermeßliche Elend der eigenen Existenz und für das Erlebnis der eigenen Vernichtung geworden war. ${ }^{24}$

Viele von Mengeles Kollegen hatten nach dem Krieg Selbstmord begangen oder waren von den Alliierten verurteilt und zum Teil auch hingerichtet worden; sie waren als tatsächliche Täter nicht mehr verfügbar. Mengele dagegen hatte nicht zuletzt seine geglückte Flucht bald zu einem der prominentesten NS-Verbrecher überhaupt gemacht. Binnen kurzer Zeit wurde er für viele, nicht nur für Überlebende, zu einer Projektionsfläche, zum „Prototyp eines SS-Arztes“25. Dieser Entwicklung konnten sich auch Zeugen wie Dr. Martina Puzyna, die als Anthropologin für Mengele Zwillinge vermessen mußte, nicht entziehen. 1972 noch mußte sie die Faszination eingestehen, die die Möglichkeit, Zwillinge in noch nie gekanntem Umfang anthropologisch vermessen zu können, auch auf sie selbst ausgeübt hatte: „Auch bei kritischer Würdigung muß ich eingestehen, daß die Ergebnisse dieser Arbeit für die Wissenschaft der Anthropologie unstreitig von höchstem Wert waren." 26 Zwar darf dies mit Recht bezweifelt werden und zeugt sicherlich von dem Drang nach Selbstrechtfertigung der Anthropologin, die Mengele bei seinen Experimenten helfen mußte und in den Versuchen wenigstens nachträglich einen Sinn sehen wollte; bezeichnend jedoch ist, daß auch sie einige Jahre später „Mengele gewissermaßen für das Abbild des Manischen" hielt, der völlig absurde Forschungen angestellt habe und „am Ende“ sogar „seine eigene Mutter umgebracht [bätte], wenn ihm das weitergeholfen hätte“27. Josef Mengele wurde, je länger die Suche nach ihm dauerte und je erfolgloser sie war, mehr und mehr zum „SS-Monster schlechthin“28.

24 Vgl. Völklein, Mengele, S. 30 f., und Zofka, KZ-Arzt, S. 259 f.

25 Langbein, Menschen, S. 385. Der 1995 verstorbene Hermann Langbein war selbst Häftling in den KL Dachau und Auschwitz, später Generalsekretär der in Wien ansässigen Häftlingsorganisation Comité d'Auschwitz.

26 Sta F/M, Az 4 Js 340/68, Erm.A., Bd. XIII, Bl. 12-19, Aussage Martina Puzyna, 31. 10. 1972.

27 Martina Puzyna im Gespräch mit Gerald L. Posner und John Ware, zit. nach: Posner/Ware, Mengele, S. 65.

28 So die Zusammenfassung dieser Entwicklung bei Klee, Auschwitz, S. 487. 


\section{Mythos Mengele}

„Mengele wurde von keiner Organisation betreut, er bewegte sich zuletzt in einem Milieu, das weit unter seinem nach Herkommen und Ausbildung angemessenen Niveau lag, er hatte nur mit wenigen Menschen Kontakt, von diesen waren nur ganz wenige über seine Identität orientiert, er hatte in der Heimat einen engen, aber festgefügten Freundes- und Unterstützerkreis, der ihn abschirmte, Kontakt hielt und ihn finanziell unterstützte. "29 Dies war, so die Staatsanwaltschaft Frankfurt 1986 in ihrem Schlußbericht, einer der Gründe, warum Josef Mengele nie gefaßt werden konnte: Der Josef Mengele, den Justiz, Medien und Nazijäger jahrzehntelang gejagt hatten, war ein völlig anderer. ${ }^{30}$

Seit Anfang der 1960er Jahre war Mengele einer der gesuchtesten NS-Verbrecher, seit $1973^{31}$ wohl der meistgesuchte überhaupt. Dennoch war er jahrzehntelang nicht aufzufinden, obwohl Nazijäger wie Simon Wiesenthal, Tuviah Friedman oder das Ehepaar Serge und Beate Klarsfeld immer wieder medienwirksam verkündeten, sie seien Mengele dicht auf den Fersen; ähnliches gilt für eine ganze Reihe von Journalisten. Im Laufe der Zeit entstand so aus einer Vielzahl von Legenden allmählich ein nahezu undurchdringlicher Mythos, der die Person Mengele umgab.

Simon Wiesenthal ${ }^{32}$ veröffentlichte 1967 ein Buch unter dem Titel „Doch die Mörder leben“, in dem er seine Jagd auf verschiedene NS-Verbrecher beschrieb; Teil dieses Buches war unter dem Titel „Der Mann, der blaue Augen sammelte“33 auch ein Kapitel zu Josef Mengele, das eine ganze Reihe „guter Storys“, aber aus heutiger Perspektive nur wenig Richtiges enthielt. Beispielhaft sei hier nur eine dieser Episoden ausführlich kommentiert ${ }^{34}$ : Im April 1961, so berichtet Wiesenthal, habe ihn ein älterer Deutscher aufgesucht und ihm erzählt, daß Bekannte, die gerade aus Ägypten zurückgekehrt seien, dort Mengele gesehen hätten. Der damalige Staatschef Nasser habe die Auswirkungen gefürchtet, falls bekannt würde, daß Ägypten Mengele Asyl gewähre, und hätte deshalb angeordnet, ihn des Landes zu verweisen. Deswegen hätte eine Gruppe alter Nazis, „die solche heiklen Angelegenheiten erledigt", eine Jacht gemietet und Mengele und seine Frau nach Kythnos gebracht,

29 Sta F/M, Az 4 Js 340/68, Schlußvermerk, 14. 7. 1986.

30 Die Mengele-Legenden, die sich bis Ende der 1960er Jahre gebildet hatten, finden sich wieder bei: Wiesenthal, Mörder, S. 193-219, und Brochdorff, Flucht, S. 123-133.

31 Am 8. 12. 1972 wurde in Berlin ein Skelett gefunden, das nahezu zweifelsfrei als das Martin Bormanns identifiziert werden konnte. Im April 1973 wurde Bormann deshalb offiziell für tot erklärt. Die vielen Bormann-Sichtungen, die in den Jahren zuvor vor allem aus Südamerika gemeldet worden waren, waren damit widerlegt.

32 Simon Wiesenthal war, wie oben bereits angemerkt, nicht der einzige unter den stark rivalisierenden Nazijägern, der falsche Mengele-Fährten verfolgte; er war allerdings der erfolgreichste, was Publizität und Medieninteresse anging. Beate Klarsfeld etwa hatte noch wenige Tage vor der Exhumierung von Mengeles Leiche in Paraguay demonstriert und Diktator Stroessner beschuldigt, Mengele immer noch zu decken. Die Reihe der Beispiele ließe sich nahezu beliebig fortsetzen. Vgl. Posner/Ware, Mengele, S. 378.

33 Wiesenthal, Mörder, S. 193-219.

34 Vgl. ebenda, S. 200-203. 
„eine kleine griechische Insel in der Nähe von Kreta“"35. Allerdings wolle Mengele sich dort nicht lange aufhalten, Eile sei also geboten.

Er selbst, Wiesenthal, sei „gerade im Begriff gewesen, nach Jerusalem zu fahren, um an der Verhandlung gegen Eichmann teilzunehmen" 36 , und somit leider verhindert gewesen. Deshalb habe er den ihm bekannten Herausgeber einer großen deutschen Illustrierten informiert, welche auch umgehend einen Reporter nach Kythnos geschickt habe. Auf der Insel habe es nur zwei große Gebäude gegeben, ein „Mönchskloster und eine kleine Wirtschaft in der Nähe des Hafens" 37 , und der Journalist sei bei seinen Nachforschungen auch prompt erfolgreich gewesen: Auf die Frage, ob er in letzter Zeit Gäste gehabt habe, habe der Gastwirt bestätigt, daß er „einen Deutschen und seine Frau“ beherbergt hätte, die aber am Tag zuvor abgereist seien. Wie das denn gehen könne, habe der Reporter sich erkundigt, wo doch an diesem Tag gar kein Schiff gekommen sei? „Eine weiße Jacht“, so die Antwort des Wirtes, „legte im Hafen an. Der Deutsche und seine Frau gingen an Bord, und die Jacht lief wieder aus, in westlicher Richtung. "38 Anschließend habe der Reporter dem Wirt und „zwei Mönche[n], die gerade daherkamen“, einen Packen Fotos gezeigt - „ohne Zögern“ hätten sie nach einem Bild von Mengele gegriffen und bestätigt, „daß dieser Mann bis gestern dagewesen sei“39.

Tatsächlich war Mengele weder in Ägypten noch auf Kythnos oder einer anderen griechischen Insel. Möglicherweise hat Wiesenthal einen Tip bekommen, denn er leitete entsprechende Informationen im Juli $1960^{40}$ an die Zentrale Stelle der Landesjustizverwaltungen zur Aufklärung von nationalsozialistischen Gewaltverbrechen in Ludwigsburg weiter - nicht erst im April 1961, und damit fast ein Jahr früher, als er in seinem Buch behauptet. Daraus folgt widerum, daß Wiesenthals Behauptung falsch ist, er sei an der Verfolgung gehindert gewesen, weil er „an der Verhandlung gegen Eichmann" 41 teilnehmen wollte. Der Eichmann-Prozeß begann in Jerusalem tatsächlich im April 1961, und es scheint, als ob Wiesenthal auf der Suche nach einem geeigneten Termin gewesen wäre, um plausibel zu erklären, $\mathrm{da} ß$ er nicht höchstselbst die Fährte des Massenmörders verfolgt hatte. Die Zentrale Stelle informierte die damals noch zuständige Freiburger Staatsanwaltschaft telefonisch von dem Anliegen Wiesenthals und verwies diesen an die zuständige Behörde weiter. ${ }^{42}$ Dort hörte man allerdings erst fast zwei Monate später, Mitte September 1960, brieflich von dem Nazijäger. ${ }^{43}$ Egal, ob Wiesenthal nun selbst einer falschen Fährte

35 Ebenda, S. 202.

36 Ebenda.

37 Ebenda, S. 203.

38 Ebenda.

39 Ebenda.

$40 \mathrm{Vgl}$. Sta F/M, Az 4 Js 340/68, Erm.A., Bd. IV, Bl. 859, Aktennotiz betr. Anruf der Zentralen Stelle, 22.7. 1960.

41 Wiesenthal, Mörder, S. 202.

42 Vgl. Sta F/M, Az 4 Js 340/68, Erm.A., Bd. IV, Bl. 859, Aktennotiz betr. Anruf der Zentralen Stelle, 22. 7. 1960.

43 Vgl. ebenda, HandA., Bd. I, Bl. 202 f., Brief Wiesenthals an die Staatsanwaltschaft Freiburg i.Br., 13. 9. 1960. Einige Tage nach Wiesenthals Brief suchte außerdem Hermann Langbein, Generalsekretär des Wiener Auschwitz-Komitees, die Freiburger Staatsanwaltschaft auf: Auch ihn habe Wiesenthal informiert, und das Komitee wolle nun einen Mittelsmann schicken, um Mengeles Spur aufzunehmen. Leider fehle das Geld, gebraucht würden etwa 
aufgesessen war oder nicht, er muß zu diesem Zeitpunkt bereits gewußt haben, daß Mengele nicht auf Kythnos gewesen war. Denn Ottmar Katz, der Reporter, der damals nach Kythnos reiste, erinnert sich an einen der Darstellung Wiesenthals in seinem Buch diametral entgegengesetzten Ablauf der Ereignisse: „Nicht ein einziges Detail [...] war korrekt. Ich brachte vier oder fünf Tage auf Kythnos zu. [...] Ein Kloster gab es nicht. Ich hielt mich zwei Tage beim örtlichen Friedensrichter auf [...], und wir gingen das Register des einzigen Hotels durch, und der einzige Name, den wir für prüfenswert hielten, gehörte, wie sich herausstellte, einem Lehrer aus München." 44 Sein Fazit: „Mengele war mit Sicherheit nicht da. [...] Ich erklärte Wiesenthal, daß das alles nicht stimmte, und dann las ich sieben Jahre später sein Buch, und da schreibt er, wir hätten Mengele um ein paar Stunden verfehlt. " 45 Als Wiesenthal sich erstmals mit der zuständigen Freiburger Staatsanwaltschaft in Verbindung setzte, wußte er offensichtlich seit mehreren Wochen von Katz, daß Mengele nie auf Kythnos gewesen war. Der Staatsanwaltschaft gegenüber verschwieg er den Mißerfolg des Reporters, und so wurden das Bundesjustizministerium und das Auswärtige Amt eingeschaltet und die griechischen Behörden auf diplomatischem Wege um Amtshilfe gebeten. Der Notenwechsel zwischen der Deutschen Botschaft in Athen und dem griechischen Außenministerium zog sich bis zum 12. Dezember 1960.46 Daß Kythnos nicht einmal, wie Wiesenthal schrieb, in der Nähe von Kreta, sondern nur wenig südöstlich von Athen liegt, ist da nur noch ein kleiner, wenn auch bezeichnender Beleg für die Genauigkeit im Umgang mit den Fakten.

Wiesenthals Ende der Geschichte: „Spanische Freunde haben Mengele und seine Frau auf ihrer Jacht mitgenommen. Der Mann hat überall Freunde. “47 Das war die Quintessenz des Mengele-Mythos, der sich nun langsam herauszubilden begann und bald immer schneller wucherte. Wiesenthal hat zur Entstehung dieses Mythos nicht unerheblich beigetragen, und dies ist ihm, trotz seiner ansonsten sicherlich großen Verdienste, durchaus zu Recht zum Vorwurf gemacht worden. ${ }^{48} \mathrm{Um}$ bei

5000 DM. Der Freiburger Staatsanwalt ließ beim Landeskriminalamt nachfragen, ob die Mittel zur Verfügung gestellt werden könnten. Das LKA verwies auf den Dispositionsfonds des Bundeskanzlers, und Langbein wollte den Zentralrat der Juden in Deutschland bitten, im Kanzleramt vorstellig zu werden. Vgl. ebenda, Bl. 208f., Aktennotiz betr. Besuch Langbeins, 29. 9. 1960.

44 Angaben von Ottmar Katz, zit. nach: Posner/Ware, Mengele, S. 255. Vgl. außerdem: „Sechs Millionen, da kann ich nur lachen“, in: Der Spiegel, 22. 4. 1985. Auch die griechische Polizei hatte "darauf hingewiesen, daß es auf der Insel Kythnos kein Kloster gebe" und nachgefragt, „ob es sich nicht um die Insel Tinos handeln könne, auf der sich ein bekannter griechischer Wallfahrtsort befinde. Die Botschaft hat vorsorglich darum gebeten, sofort in Tinos nachzuforschen." Sta F/M, Az 4 Js 340/68, HandA., Bd. II, Bl. 82v, Mitteilung der Deutschen Botschaft in Athen an das Auswärtige Amt, 2. 12. 1960.

45 Ebenda.

$46 \mathrm{Vgl}$. Sta F/M, Az 4 Js 340/68, HandA., Bd. II, Bl. 10 und 14, Briefe des Justizministeriums Baden-Württemberg an den Bundesminister der Justiz, 6. 10. 1960 und den Generalstaatsanwalt in Karlsruhe, 14. 10. 1960; Bl. 15, Brief des Bundesministers der Justiz an das Justizministerium Baden-Württemberg, 12.10. 1960; Bl. 69 und 84, Noten des Griechischen Außenministeriums an die Deutsche Botschaft in Athen, 11.11. und 12.12. 1960; Bl. 76, 77 und $82 \mathrm{v}$, Mitteilungen der Deutschen Botschaft in Athen an das Auswärtige Amt, 22.10, 4.11. und 2.12. 1960.

47 Wiesenthal, Mörder, S. 203.

48 Vgl. auch die beiden Wiesenthal-Biographien von Hella Pick und Allan Levy: Pick, Wiesenthal, und Levy, Akte. 
dem Beispiel von Mengeles Kythnos-Aufenthalt zu bleiben: Bald nach dem Erscheinen von Wiesenthals Buch war in einer anderen Darstellung aus der Flucht aus Ägypten auf eine kleine griechische Insel bereits „eine ausgedehnte Reise durch die griechische Inselwelt“ geworden, auf der ihn der „Reporter einer bekannten Illustrierten verfolgt, aber nie gefunden hat"; denn „immer war Mengele eine Nasenlänge voraus" 49 . Dies habe aber durchaus nicht an Mengeles Findigkeit gelegen vielmehr hätten die Führer des „Römischen Weges“, einer Nazi-Fluchthilfe-Organisation, zum Schutz Mengeles „zwei in den Nahen Osten emigrierte ehemalige SD-Leute abgestellt" 50 .

In dem argentinischen Kurort Bariloche soll er 1960 eine Jüdin getroffen haben, die er an der Tätowierung als ehemaligen Auschwitz-Häftling erkannte; wenige Tage später sei sie von einer Bergwanderung nicht zurückgekehrt - sie soll eine Agentin des israelischen Geheimdienstes gewesen und Mengele von einem geheimen Nazi-Netzwerk gewarnt worden sein. ${ }^{51} 1964$ soll Mengele im Hotel „Tyrol“ in Paraguay nur knapp entkommen sein: Simon Wiesenthal schreibt, das Hotel „in der Nähe von Hohenau“ sei „ein beliebter Treffpunkt der dortigen guten Gesellschaft" gewesen und sogar „General Stroessner hält sich dort von Zeit zu Zeit über das Wochenende auf“. Es sei „eine dunkle, schwüle Nacht“ im Sommer 1964 gewesen, als das „Komitee der Zwölf“ (eine Gruppe von jüdischen Holocaust-Überlebenden) Mengele in Zimmer 26 dieses Hotels aufgespürt habe; er sei aber kurz zuvor telefonisch gewarnt worden und habe „das Hotel zehn Minuten vor ihrem Eintreffen verlassen“. Die Flucht sei Hals über Kopf erfolgt, so „daß er sich nicht einmal die Zeit nahm, den Pyjama auszuziehen, sondern den Straßenanzug darüberzog “.52 Auch diese Geschichte war offensichtlich ausbaufähig: Michael Bar-Zohar bietet einige weitere Details: Man habe am nächsten Morgen einen der Juden erschossen aufgefunden; außerdem habe das Entführungskommando geplant, Mengele auf eine Jacht an der Küste zu entführen und ihn dort hinzurichten. ${ }^{33}$ Tatsächlich hatte das Hotel Tirol kein Zimmer 26 und nicht einmal Telefon ${ }^{54}$, Paraguay hat keine Küste und Mengele hatte das Land schon vier Jahre zuvor verlassen.

Aufgehalten haben soll sich Mengele entweder in einer paraguayanischen Grenzfestung, deren Zufahrtsstraßen „von Militär- und Polizeistreifen schärfstens überwacht" würden. Diese Posten hätten strikten Befehl, „auf Eindringlinge sofort zu schießen“, und zusätzlich halte er sich „auf eigene Kosten vier schwer bewaffnete Leibwächter" 55 . Daneben gab es noch eine ganze Reihe anderer angeblicher Aufenthaltsorte. So wurde etwa auch behauptet, er halte sich im tiefen Urwald nahe der brasilianischen Stadt Eldorado verborgen; dort habe er einen riesigen Besitz erworben in einem Gebiet, in dem sich auch Martin Bormann versteckt halte und das für Außenstehende praktisch nicht zu erreichen sei. Dies liege vor allem

49 Brochdorff, Flucht, S. 129.

50 Ebenda.

51 Wiesenthal, Mörder, S. 199f.; Bar-Zohar, Avengers, S. 239 f., und Brochdorff, Flucht, S. 129 f.

52 Wiesenthal, Mörder, S. $211 \mathrm{f}$.

53 Vgl. Bar-Zohar, Avengers, S. 245 f.

54 Vgl. Posner/Ware, Mengele, S. 263.

55 Wiesenthal, Mörder, S. 219. Wiesenthal war von diesen Angaben auch 1988 noch wider besseres Wissen überzeugt: vgl. die fast wörtlich identische Passage in Wiesenthal, Recht, S. 146. 
daran, daß dort „bezahlte Guaraní-Indianer [...] für manche Überraschungen“56 sorgten.

Mengele wurde zu einem Phantom mit besten Kontakten, der wie die Spinne in einem Netz aus alten Nazis saß und die Fäden zog, den seine Informanten vor jedem Schritt der Gegenseite warnten, der - natürlich - auch vor Mord nicht zurückschreckte und der umgeben von einer Schar von Leibwächtern unter dem Schutz Alfredo Stroessners in Paraguay vermutet wurde. ${ }^{57}$ Unzählige Journalisten und Abenteurer wollten ihn im Laufe der Jahre ausfindig gemacht und Interviews geführt ${ }^{58}$, ihn fotografiert oder gefilmt haben. 1966 etwa gelang dem tschechischen Reporter Adolpho Cicero eine 3,5 Sekunden lange Filmaufnahme eines Mannes, den er für Josef Mengele hielt. Cicero hatte den Gesuchten nie gesehen und verfügte lediglich über wenige, jahrzehntealte Fotos. Der Mann, den Ciceros Aufnahmen zeigen, war jedenfalls nicht Mengele, und dennoch gingen die Bilder um die Welt und wurden in vielen Zeitungen abgedruckt - zum Teil bis heute. ${ }^{59}$ Immer wieder kam es zu angeblichen Mengele-Sichtungen, und auch die seriösesten Blätter waren sich für die Mengele-Sensationsmeldungen nicht zu schade. So saß etwa die Londoner Sunday Times 1967 dem Ex-Nazi Erich Karl Wiedwald auf, der behauptete, Mengele sei im Majorsrang in die paraguayanische Armee eingetreten. Dies wisse er aus seinen Tagen als Leibwächter Martin Bormanns, der sich einer Gesichtsoperation unterzogen habe und mit Magenkrebs im Sterben liege. Obwohl schnell klar war, daß Wiedwald log, hielten sich seine Mengele betreffenden Behauptungen hartnäckig. ${ }^{60}$ Bisweilen meldeten sich auch Leute, die den KZ-Arzt sogar getötet ${ }^{61}$ haben wollten. Das wohl bekannteste Beispiel ist Erich Erdstein, ein ehemaliger

56 Brochdorff, Flucht, S. 133. Vgl. auch Bar-Zohar, Avengers, S. $246 \mathrm{f}$.

57 Vgl. auch die Autobiographie Benno Weiser Varons, der von 1968-1972 israelischer Botschafter in Paraguay war: Weiser Varon, Professions, S. 360-388. Weiser Varon ist einer der Kritiker Wiesenthals, und obwohl auch er 1983 noch fest davon überzeugt war, Mengele halte sich in Paraguay auf, wies er auf die Möglichkeit hin, daß Mengele möglicherweise "the unenviable life of the hunted" führe und "that my intuition tells me that Mengele's sole punishment is that instead of living in the luxury and beauty that as the rightful heir to his family enterprise he could afford, he has had to spend his last 25 years in a remote and backward semi-jungle“. Außerdem müsse man damit rechnen, daß „one of these days Mengele were to die, peacefully in bed" (Weiser Varon, Living).

$58 \mathrm{Vgl}$. z. B. Bormans Gang: World exclusive challenge! To Nazi-hunter Simon Wiesenthal ... Now follow my steps to the Doctor of Death, in: Daily Express, 1. 12. 1972 und Bormanns Gang. I find the Nazi Doctor of Death, ebenda. Die beiden Artikel stammten von Ladislas Farago, der in seinem Buch „Aftermath. Martin Bormann and the Fourth Reich“ später behauptete, er habe Mengele getroffen und mit ihm gesprochen. Vgl. Weiser Varon, Professions, S. $383 \mathrm{f}$.

59 Vgl. Sta F/M, Az 4 Js 340/68, Erm.A., Bd. V, Bl. 1213-1215, Telegramm an die Sta Freiburg im Breisgau betr. Filmaufnahmen Ciceros, 1. 2. 1967, und Posner/Ware, Mengele, S. $264 \mathrm{f}$.

Diese Bilder sind bis heute nicht verschwunden, obwohl deutlich zu erkennen ist, daß die abgebildete Person nicht Mengele ist, wenn man die Abbildung mit authentischen MengeleFotografien vergleicht. Der Spiegel etwa verwendet bis heute ein solches falsches MengeleBild: vgl. Im Visier der Nazijäger, in: Der Spiegel, 3. 9. 2001. Das dort verwendete Bild findet sich bereits 1967, in: Spur nach Eldorado, in: Der Spiegel, 19. 6. 1967, S. 97. Auf einen entsprechenden Hinweis erhielt der Verf. von der Redaktion leider keine Antwort.

60 Wiedwald hatte behauptet, SS-Gruppenführer Glücks gesehen zu haben, der während des Kriegs für die Konzentrationslager zuständig gewesen war. Der hatte sich aber schon 1945 in britischer Gefangenschaft das Leben genommen. Vgl. Posner/Ware, Mengele, S. 266.

61 Vgl. ebenda, S. 266-268. 
brasilianischer Polizist, dem es gelang, mehrere Zeitungen davon zu überzeugen, daß er Mengele im September 1968 getötet habe. Er habe ihn während des Versuchs gefangengenommen, einen Grenzfluß nach Paraguay zu überschreiten. Wenig später sei dann aber ein paraguayanisches Schnellboot erschienen und habe umgehend das Feuer eröffnet. Als sein eigenes Boot von mehreren Männern mit Maschinenpistolen geentert worden sei, habe er Mengele erschossen, der, von Kugeln in Brust und Nacken getroffen, über Bord gegangen sei. Als Posner und Ware Erdstein später mit der Tatsache konfrontierten, daß Mengele erst 1979 beim Baden ertrunken war, erklärte er lapidar, daß er dann wohl einen Doppelgänger erschossen habe.

Trotz internationaler Aufmerksamkeit, die sich in einer Fülle von Schlagzeilen manifestierte, entkam der KZ-Arzt scheinbar immer wieder, was die ungezählten Verschwörungstheorien nur zu bestätigen schien. Auch Kunst und Unterhaltungsindustrie entdeckte die Figur des KZ-Arztes für sich: Die diabolische Figur des „Doktors“ in Rolf Hochhuths Theaterstück „Der Stellvertreter" trägt unverkennbar die Züge Josef Mengeles ${ }^{62}$ und Hollywood vereinnahmte den KZ-Arzt in zwei durchaus erfolgreichen Kinofilmen („The Boys from Brazil“63 und „Marathon Man" ${ }^{64}$ ), in denen Mengele-inspirierte Charaktere die Hauptrolle spielten. Der Mythos Mengele wurde zum Medienereignis, und er scheint bisher nichts an negativer Faszinationskraft verloren zu haben. ${ }^{65}$

$62 \mathrm{Vgl}$. Hochhuth, Stellvertreter.

63 The Boys from Brazil, Spielfilm, Regie: Franklin J. Schaffner, UK/USA 1978, basierend auf dem gleichnamigen Buch von Ira Levin. Der Nazijäger Lieberman (Vorbild: Simon Wiesenthal) findet heraus, daß Josef Mengele im brasilianischen Urwald 94 Hitler-Klone erschaffen und weltweit verteilt hat. Nun versucht der ehemalige KZ-Arzt, durch eine möglichst genaue Simulation der jeweiligen Lebensumstände nicht nur physisch, sondern auch psychisch einen neuen Hitler zu erschaffen, was u.a. dazu führt, daß die jeweiligen „Väter" ermordet werden, sobald die Klone das Alter erreicht haben, in dem auch Hitler seinen Vater verlor.

64 Marathon Man, Spielfilm, Regie: John Schlesinger, USA 1976. Hier war Mengele das Vorbild für den KZ-Arzt Szell (gespielt von Sir Laurence Olivier), der in einen großangelegten Diamantenschmuggel verwickelt ist. Bekannt wurde vor allem eine Szene, in der Szell seinen Gegenspieler, einen jüdischen Studenten, dessen Bruder in dem Fall ermittelte und ermordet wurde, durch eine zahnärztliche „Behandlung“ foltert.

$65 \mathrm{Vgl}$. Nichts als die Wahrheit, Spielfilm, Regie: Roland Suso Richter, BRD 1999, in dem sich Götz George als Josef Mengele der Justiz stellt, um seine Auffassung von der Wahrheit vor der Welt vertreten und in einer zynischen Argumentationskette darlegen zu können. 